\title{
17 Sklaverei und der Topos des krimtatarischen Kriegers
}

\begin{abstract}
Die Tataren führen gewöhnlich Krieg gegen die Polen, die Bewohner der Rus', Moskaus, die Čerkessen, Moldauer und Ungarn. Viele der Gefangenen, die sie diesen Völkern abnehmen, machen sie zu Sklaven. Eine andere Beschäftigung außer der Kriegsführung kennen sie nicht. ${ }^{1}$
\end{abstract}

Diese aus der Feder eines Dominikanermönchs mit Namen Jean de Luc stammende Beschreibung der Krimtataren wurde im Jahre 1625 verfasst. Sie zeichnet sich durch eine gewisse Überzeitlichkeit aus, zog sich doch die Vorstellung, dass die Krimtataren keine „andere Beschäftigung außer der Kriegsführung kennen“, und dass das Krim-Chanat ein auf die Kosten der Anrainer lebender Parasitenstaat sei, bis in das 20. Jahrhundert: „Die hauptsächliche Beschäftigung der Krimtataren waren der Krieg und Raubzüge, die Plünderung und Gewinn zum Ziel hatten“2, so war beispielsweise in der "Großen Sowjetischen Enzyklopädie“ (Bol’šaja Sovetskaja Ėnciklopedija, BSĖ) aus dem Jahre 1953 zu lesen. Diese negative Einschätzung einer Nationalität, welcher immerhin in den 1920er Jahren innerhalb der UdSSR im Rahmen einer Autonomen Sozialistischen Sowjetrepublik (ASSR) begrenzte Sonderrechte zugestanden worden waren, mag erstaunen, erklärt sich aber durch die Ereignisse im Zweiten Weltkrieg. Die krimtatarische Bevölkerung war im Mai 1944 nach Zentralasien deportiert und die ASSR 1945 aufgelöst worden, da ihre muslimischen BewohnerInnen von Stalin unter den Kollektivverdacht gestellt worden waren, sie hätten im Weltkrieg mit den nationalsozialistischen Besatzern kollaboriert. Die KrimtatarInnen hatten in der Nachkriegszeit in der UdSSR entschieden keine Lobby (Kapitel 35).

In beiden Zitaten wird noch etwas anderes deutlich, nämlich dass sowohl der Mönch de Luc als auch der namentlich nicht erfassbare Autor des Eintrags über die Krimtataren in der „Großen Sowjetischen Enzyklopädie“ nicht an Krimtatarinnen dachten, als sie über das Wesen dieser Gruppe sinnierten. In diesen und anderen Beschreibungen galt zumeist Folgendes: „Der idealtypische Krimtatare war [...] männlichen Geschlechts, hatte sich über lange Zeit mehr oder minder ausschließlich mit Krieg befaßt [und] war der Ordnung und Sauberkeit nicht sehr zugeneigt. “' In der Wahrnehmung der meisten AutorInnen aus dem Norden waren Krimtataren Krieger und besaßen zumindest ein Pferd, um das ein jeder Tatare

1 Opisanie (1879), 470.

2 Krymskie Tatary (1953).

3 Jobst (2007b), 206. Vgl. zum männlichen Blick auf die krimtatarische Bevölkerung 206-218.

๑ OpenAccess. ( 2020 Kerstin S. Jobst, publiziert von De Gruyter. (cc))BY Dieses Werk ist lizenziert unter der Creative Commons Attribution 4.0 International. https://doi.org/10.1515/9783110520620-019 
sich, so noch einmal de Luc, mehr kümmere „als um sich selbst.“4 Der polnische Diplomat Broniewski widmete dem Thema Tataren und Pferde sogar einen eigenen Abschnitt, in dem er sich anerkennend über die Qualität dieser Tiere äußerte: „Alle [Krimtataren] benutzen tatarische Pferde nach ihrem Bedarf, die elegant, sehr gut, kräftig, schnell, mittelhoch und sehr bequem sind und alle Mühe aushalten."

Dass sich nicht-muslimische AutorInnen über lange Zeit viel mehr mit krimtatarischen Kriegern befassten als beispielsweise mit krimtatarischen Bauern, hatte einen einfachen Grund: Die Kontakte zwischen dem Krim-Chanat (in Verbund mit dem Osmanischen Reich oder auch alleine) und seinen nördlichen Nachbaren waren über fast dreihundert Jahre überwiegend kriegerischer Natur, und diese ,Erfahrung wirkte lange im kollektiven Bewusstsein von PolInnen, RussInnen oder UkrainerInnen nach. Ohnehin, also nicht nur in Bezug auf die KrimtatarInnen, hat sich durch die jahrhundertelange wechselhafte ostslavischmuslimische Beziehungsgeschichte ein spezifisches MuslimInnenbild im russischen Kontext herausgebildet, in dem das Bild des Kriegers eine große Rolle spielte. ${ }^{6}$ Da das krimtatarische Heer im Wesentlichen aus Kavalleristen bestand, prägte sich der Topos des reitenden Krimtataren besonders ein. Broniewski beschrieb dieses Phänomen, welchem ein Moment der Täuschung, der Kriegslist innewohnte, eindringlich wie folgt:

Sehr viele in ihrem tatarischen Heer sind durchaus unkriegerisch und waffenlos, und diese führen wegen des zahlreichen Heeres beinah unendlich viele Pferde mit in den Krieg - denn auch ein Tatar von niedrigstem Stande gibt sich nicht mit einem oder zwei, ja nicht mit drei oder vier und mehr Pferden, die er eben an der Hand mit sich führen kann, zufrieden. Darum erscheint das Tatarenheer so zahlreich, riesig und gar furchtbar, das - wenn es aus der Ferne erblickt wird - von unseren Leuten für ein ziemlich gewaltiges und zahlreiches und bedeutendes Heer gehalten wird. ${ }^{7}$

Nicht alle Kontakte zwischen dem Krim-Chanat und Moskau oder Polen-Litauen waren aber kriegerischer Natur. Häufig fungierten die Chane auch als Mittler zwischen dem Kreml und der Hohen Pforte: Als etwa russische Kaufleute den Handel mit den Küstenstädten des Schwarzen Meeres intensivieren wollten und dafür gute Beziehungen mit den Osmanen notwendig waren, stießen sie auf konkrete Probleme. Der Weg nach Istanbul war lang, das osmanische Hofzere-

4 Opisanie (1879), 480.

5 Broniovius (2011), 119.

6 Vgl. Jobst (2011a).

7 Broniovius (2011), 119. 
moniell komplex und fremd, was den Austausch von Gesandtschaften schwierig machte. Schon deshalb wandten sich die mit dem Osmanischen Reich handelnden russischen Kaufleute noch im 16. Jahrhundert an Bağçasaray und baten um Vermittlung in Handelsdingen. ${ }^{8}$

Das Schwarze Meer und die Halbinsel Krim hatten wegen ihrer geographischen Lage eine wichtige Mittlerfunktion: Zwischen Peripherie und Metropole bzw. zwischen Steppe und Küste gelegen, war hier seit der Antike ein bedeutender Umschlagplatz für Waren aller Art gewesen - und der zeittypische und kulturübergreifende Menschenhandel war ein bedeutender Wirtschaftszweig. ${ }^{9}$ Bei der flüchtigen Lektüre zeitgenössischer oder auch aktuellerer Darstellungen wie die de Lucs oder der BSÉ gewinnt man allerdings den Eindruck, der Sklavenhandel sei ausschließlich das Metier der Krimtataren, zuweilen im Verbund mit dem Osmanischen Reich, gewesen. Die Opfer dieser Profession seien entsprechend ausnahmslos die slavische bäuerliche Bevölkerung in dem der Halbinsel vorgelagerten Steppengürtel, dem sog. Wilden Feld (russ. Dikoe Pole; poln. Dzikie Pole; ukr. Dike Pole) gewesen, das somit zu einer unsicheren, für die dauerhafte Besiedlung und Bewirtschaftung unbrauchbaren Grenzregion geworden sei. Diese Einschätzung ist keineswegs von der Hand zu weisen, denn es ist unstrittig, dass krimtatarische Reiterarmeen beinahe jährlich gen Norden zogen, um dort Gefangene und Beute $\mathrm{zu}$ machen. ${ }^{10}$ Ökonomisch waren die fruchtbaren Gebiete sowohl für Polen-Litauen als auch für den Moskauer Staat somit nur eingeschränkt nutzbar und erforderten zudem umfängliche Grenzsicherungen. Während im letzteren Fall ein unumschränkter zarischer Herrscher die Sicherungsmaßnahmen ohne Widerstand anordnen konnte (auch wenn diese keinesfalls immer erfolgreich waren), gelang dies in Polen-Litauen nicht so einfach. Der König stand dort sehr selbstbewussten Adligen gegenüber, die „sich in Durchsetzung ihrer Partikularinteressen weitgehend den Forderungen“ nach finanzieller Beteiligung entzogen. ${ }^{11}$ Und nach der Union von Lublin 1569 oblag die Sicherung der südlichen Grenzen ohnehin allein den Herrschern, die fortan den polnischen Juden spezielle Steuern auferlegten, welche die Kosten für die Grenzsicherung aber nicht abdecken konnten. ${ }^{12}$ Die Einfälle der bewaffneten Reiter aus dem Süden verschärften überdies ein Problem, an dem alle europäischen Staaten in der Frühen Neuzeit litten - den Bevölkerungsmangel, der durch

8 Vgl. dazu Kusber (1998).

9 Vgl. hierzu auch die Hinweise in den Kapiteln 3 und 5.

10 Vgl. hierzu die Aufstellung über „Tatar Raids for Captives“ von 1468 bis 1694 bei Fisher (1972b), 580-583.

11 Jobst (2011b), 21.

12 Skorupa (1994), insbesondere 263 und 285. 
einen geringen Geburtenüberschuss, Naturkatastrophen, Epidemien und Kriege schon ohne Sklavenbeutezüge eklatant war. Wie hoch die Zahl der dauerhaft oder zeitweise in die Sklaverei gelangten Menschen aus dem östlichen Europa war, wurde lange Zeit kontrovers diskutiert: Michael Khodarkovsky hielt 2002 beispielsweise zwischen 150.000 und 200.000 in die Sklaverei gelangte UntertanInnen allein in der ersten Hälfte des 17. Jahrhunderts für wahrscheinlich. ${ }^{13}$ Der Rückkaufswert jedes Einzelnen - der Freikauf von Gefangenen galt im Moskauer Reich Ivans IV. als Christenpflicht, für die eine spezielle Steuer eingeführt wurde ${ }^{14}$ - lag bei ca. fünf Rubel, so dass allein für den besagten Zeitraum zwischen 750.000 und 1.000.000 Rubel fällig gewesen wären. Das war eine hohe Summe und inkludierte nicht die Tribute, „Geschenke“ und Steuern, die ohnehin an das Chanat zu entrichten waren. ${ }^{15}$ In Polen-Litauen gab es übrigens keinen organisierten Rückkauf von in Gefangenschaft gelangten UntertanInnen. ${ }^{16}$ In jedem Fall war der Menschenverlust immens, gehen neuere Schätzungen doch allein für den Zeitraum zwischen 1500 und 1700 von ca. zwei Millionen Menschen aus. ${ }^{17}$ Auch für den frühneuzeitlichen Kontext ist unklar, ob der über das Schwarze Meer abgewickelte Sklavenhandel den transatlantischen übertraf. ${ }^{18}$

Ohne Zweifel war das Schicksal vieler Sklavinnen und Sklaven sehr hart. Broniewski, der ansonsten ja kein gänzlich negatives Krimtatarenbild zeichnete, ${ }^{19}$ wurde an dieser Stelle sehr deutlich: „Das Los der Gefangenen bei den Tataren ist ganz erbärmlich, denn die einfacheren werden von ihnen durch Hunger, Nacktheit und Prügel niedergedrückt, sodass sie lieber sterben als leben wollen.“20 Auch Alan Fisher kam vor einigen Jahren auf der Grundlage zeitgenössischer Berichte $\mathrm{zu}$ einer ähnlichen Einschätzung: „[O]ften in chains and always on

13 Khodarkovsky (2002), 223.

14 Vgl. Lavrov (2009).

15 Khodarkovsky (2002), $223 \mathrm{f}$.

16 Vgl. Lavrov (2009), 443, der darauf hinweist, dass allein der Vatikan und katholische Orden sich auf dem Gebiet des Gefangenenfreikaufs engagierten.

17 So das Ergebnis des polnischen Osmanisten Dariusz Kołodzieczyk: Kołodzieczyk (2006), 151. Vgl. die Diskussion der verschiedenen Forschungspositionen bei Davies B. (2007), 23-27.

18 So Kołodziejczyk (2011), xiv. Vgl. demgegenüber, dass insgesamt ca. zwölf Millionen Menschen aus dem westlichen, zentralen und südlichen Afrika als Sklaven in beide Amerikas verbracht wurden: Segal (1995), 4. Ein weiterer Hotspot des Sklavenhandels war das Mittelmeer, der dort allerdings häufig in ,privater Initiative durchgeführt wurde, nämlich durch Piraten. Vgl. dazu: Davies R. (2004). Dieser Autor schätzt, dass von 1500 bis 1800 dort zwischen einer und eineinhalb Millionen Menschen in die Sklaverei gerieten.

19 Vgl. dazu Albrecht (2011).

20 Broniovius (2011), 115. 
foot“21, starben viele von ihnen bereits auf dem Weg zum wichtigsten Sklavenumschlagplatz am nördlichen Schwarzen Meer - Kefe, das ehemalige Caffa, auf der Krim. ${ }^{22}$ Dort wurden diejenigen weiterverkauft, welche nicht fürderhin auf der Halbinsel selbst ihr Sklavendasein fristen mussten, wurde doch ein gewisser Prozentsatz vor Ort verteilt. So standen dem Krim-Chan und militärischen Führern Anteile an der ,Ware' zu, welche diese offenbar auch regelhaft beanspruchten. Broniewski beschrieb die Verteilung folgendermaßen:

Sobald er an der Grenze [des Chanats] angekommen ist, erhält der Khan vom ganzen Heer den zehnten Teil der vornehmen Gefangenen. Die Anführer der Truppen aber bekommen von den einzelnen Abteilungen, und die, die eine größere Zahl von Gefangenen herbeibringen, erhalten von den übrigen vornehme Gefangene. Die übrigen Tataren des gemeinen Volks teilen sich die Gefangenen innerhalb ihrer Truppeneinheit. ${ }^{23}$

Damit nahm, zumindest wenn wir unserem polnischen Diplomaten folgen, der Chan immerhin weniger als das obligatorische Fünftel, welches nach dem Koran Allah zugestanden hätte.

Nachdem in Kefe Zwischenhändler die übrigen Sklaven aufgekauft hatten, wurden diese auf Schiffen nach Istanbul gebracht, die unter den damaligen technischen Bedingungen mit einem Zwischenstopp zumeist in Sinope an der Nordküste des Schwarzes Meeres (heute: Sinop) ca. zehn Tage für die Überfahrt benötigten. Dort wurden sie weiterverkauft, wenn nicht ein Abgesandter des Sultans Bedarf anmeldete: Attraktive Frauen kamen häufig in den Harem des Palastes, kräftige Männer wurden zu Dienstboten - und nicht selten noch vor Ort kastriert. Viele männliche Sklaven aus dem östlichen Europa endeten auch auf den Galeeren des Sultans, wo die Todesrate traditionell sehr hoch war. ${ }^{24}$ Das Los vieler von krimtatarischen Reitertruppen gefangengenommenen Menschen aus dem östlichen Europa war - wie geschildert - unbestritten sehr hart. Allerdings waren die über das Schwarze Meer und die Krim abgewickelten Geschäfte mit Menschen keinesfalls eine tatarische oder - zumal wenn man die Rolle Istanbuls beim Weiterverkauf berücksichtigt - osmanische oder exklusiv muslimische An-

21 Fisher (1972b), 582f., dem ich auch im Weiteren überwiegend folge.

22 Kefe war der zentrale Verschiffungshafen. Primär galt für Gefangene aus dem Moskauer Staat, dass diese von Azov aus dorthin übers Meer transportiert wurden, während die aus Polen-Litauen einen Umweg über Özi/Özü, der heutigen zur Ukraine gehörenden Hafenstadt Očakiv (ukr.; russ. Očakov), nehmen mussten, bevor sie per Schiff nach Kefe gebracht wurden.

23 Broniovius (2011), 115.

24 Fisher (1972b), 584. 
gelegenheit, sondern eine internationale. ${ }^{25}$ Die institutionalisierte Sklaverei überschritt auch in damaliger Zeit die Grenzen von Imperien genauso wie die von Religionen und Kulturen. ${ }^{26}$ Bei der Betrachtung des Menschenhandels darf deshalb nicht vergessen werden, dass bereits die von den europäischen Oberschichten seit der Renaissance so verehrte Kultur der Hellenen auf einer Sklavenhalterökonomie fußte. Und mit einem deutlichen Seitenhieb gegen das westliche, vermeintlich so entwickelte römisch-katholisch geprägte Europa ätzte ein namentlich nicht genannter russischer Autor noch im Jahr 2003 gegen die am Schwarzen Meer lange einflussreichen Stadtstaaten von Venedig, Genua, Pisa und Florenz, ohne deren Zutun der Sklavenhandel nicht möglich gewesen wäre, wie er zu Recht bemerkte: Europäische Renaissance und Sklavenhandel hätten sich „Hand in Hand“" entwickelt. ${ }^{27}$ Wenn das Phänomen auch ein globales war, so war dessen Bewertung doch uneinheitlich, wie es Mikhail Kizilov auf der Grundlage von Quellen christlicher, muslimischer und jüdischer Autoren anschaulich dargelegt hat: Ohne die Sklaverei grundsätzlich in Frage zu stellen - galt diese in der Zeit doch als normale Handelsaktivität -, wurde implizit und explizit häufig unterschieden, wer Opfer und wer Täter, was akzeptabler und inakzeptabler Menschenhandel war. ChristInnen störten sich demnach besonders an der Gefangennahme ihrer Landsleute durch MuslimInnen, würden diese doch dadurch dem ihrer Meinung nach schädlichen Einfluss des Islams ausgesetzt. Zudem unterstellten sie MuslimInnen ohnehin, besonders grausam zu sein. Die unfreie Arbeit der eigenen Leibeigenen wurde indes nicht kritisiert. ${ }^{28}$ Für muslimische Autoren sind umgekehrt weniger Klagen über das Schicksal ihrer Glaubensbrüder in Gefangenschaft bekannt, zumal die Rolle der Sklaverei schon im Koran als ,common human institution“ betrachtet wurde. ${ }^{29}$ Die größten Unterschiede in den jüdischen, muslimischen und christlichen Diskursen über die Sklaverei gab es wohl hinsichtlich der Frage nach möglichen Konversionen: Während jüdische Autoren den Religionswechsel ihrer in Gefangenschaft geratenen Glaubensbrüder überwiegend strikt ablehnten, wurde dieser in den beiden anderen Fällen zuweilen als legitime Option zur Verbesserung des persönlichen Schicksals gewertet. ${ }^{30}$

Wie dem auch sei: Die Beschaffung der ,Ware', also der SklavInnen, oblag im Schwarzen Meer in aller Regel nicht den BewohnerInnen der italienischen Ko-

25 Vgl. hierzu den Sammelband Witzenrath (2016), in dem zahlreiche Beiträge den Anteil von Slaven sowohl als ,Opfer‘ als auch als ,Täter‘ behandeln.

26 Zur Geschichte der Sklaverei u.a. Lavrov (2009), 425-443.

27 Russkij Krym (2003), 1.

28 Kizilov (2007), 30.

29 Vgl. Fisher (1972b), 575.

30 Kizilov (2007), $30 \mathrm{f}$. 
lonien, die eher im Verkauf tätig waren. Schon vor der Vertreibung der Genueser durch die Osmanen im Jahr 1475 lag diese in den Händen der Tataren - und Emissäre ostslavischer Adliger aus dem Moskauer Reich lieferten ebenfalls den begehrten Handelsgegenstand aus den Reihen ihrer eigenen Landsleute auf die Sklavenmärkte von Caffa oder Tana. ${ }^{31}$ Selbst nachdem Caffa zum osmanischen Kefe geworden war, lag der Handel weiterhin nur zu einem geringen Teil in muslimischer Verantwortung. Mehrheitlich wurde dieser von Griechen, Armeniern, Italienern oder Juden durchgeführt. ${ }^{32}$ Auch Polen-Litauen und das Moskauer Reich machten ihrerseits während der osmanischen und krimtatarischen Raubzüge Gefangene - die sie entweder selbst behielten oder nach Westeuropa verkauften. ${ }^{33}$ Dementsprechend gab es ebenso in Moskau Sklavenmärkte, auf denen u.a. Menschen aus den baltischen oder deutschsprachigen Gebieten verkauft wurden, übrigens bis Mitte des 17. Jahrhunderts auch an muslimische Händler. ${ }^{34}$

Eine Unterscheidung in - im weitesten Sinne - staatliche (z. B. das KrimChanat, das Moskauer Reich) und private (griechische, armenische Händler etc.) Akteure im Sklavengeschäft oder beim Rückkauf von in Gefangenschaft geratenen Menschen im Schwarzen Meer greift zu kurz. Vielmehr gab es noch weitere handelnde Gruppen, die grundsätzlich in einem besonderen herrschaftlichen Loyalitätsverhältnis standen oder, besser, hätten stehen sollen und die sich ebenfalls in diesem Geschäft umtaten. Zu nennen sind hier insbesondere Folgende: die im Chanat ohnehin häufig selbständig agierenden $\mathrm{Clans}^{35}$, die Nogaier sowie die Zaporoger und Don-Kosaken. ${ }^{36}$ Während über die Mirza als Faktor in der Geschichte des Krim-Chanats bereits einiges berichtet worden ist, müssen Zaporoger und Don-Kosaken nicht nur im Kontext der Menschenraubzüge noch genauer betrachtet werden.

31 Fisher (1972b), 577.

32 Fisher (1972b), 583 und 584. Der Sklavenhandel in Istanbul lag nach Fisher „[w]ithout exception“ in jüdischen Händen. Vgl. aber auch die differenzierteren Aussagen zur Täter- und Opferrolle von Juden im Menschenhandel bei Kizilov (2007), 25-30.

33 Beispiele dafür bei Spuler (1965), 386.

34 Hellie (1982), 73f. und 82f. Die Unterschiede zwischen der bekanntlich erst 1861 im Zarenreich abgeschafften Leibeigenschaft und Sklaverei können hier nicht im Detail ausgeführt werden. Kurz gesagt besaßen Leibeigene eine geminderte Rechtsfähigkeit, und deren BesitzerInnen hatten ihrerseits auch gewisse rechtliche Verpflichtungen diesen gegenüber. Sklaven hingegen galten als - sprechende - Sachen. Dass es in beiden Fällen häufig Unterschiede zwischen Theorie und Praxis gab, ist unstrittig.

35 Vgl. einige Beispiele bei Fisher (1972b), 589.

361641 eroberten die Donkosaken das osmanische Azov am Unterlauf des Don und beteiligten sich Ende des 17. Jahrhunderts auf Seiten des Zarenreichs an diversen Feldzügen gegen das KrimChanat. 
